\title{
The incidence of venous thromboembolism in cervical cancer: a nationwide population-based study
}

\author{
Shiang-Jiun Tsai ${ }^{1 \dagger}$, Ying-Xu Ruan ${ }^{2}$, Ching-Chih Lee ${ }^{3,5}$, Moon-Sing Lee ${ }^{1,5}$, Wen-Yen Chiou ${ }^{1,5}$, Hon-Yi Lin ${ }^{1,5}$, \\ Feng-Chun Hsu', Yu-Chieh Su, ${ }^{4,5}$ and Shih-Kai Hung ${ }^{1,5^{*}}$
}

\begin{abstract}
Background: Venous thromboembolism (VTE) is a life-threatening condition that occurs as a complication of cervical cancer. The aim of this study was to evaluate the incidence of VTE in cervical cancer patients during a 5year follow-up.

Methods: The study analyzed data deposited between 2003 and 2008 in the National Health Insurance Research Database (NHIRD), provided by the National Health Research Institutes in Taiwan. Totally, 1013 cervical cancer patients after treatment and 2026 appendectomy patients were eligible. The Kaplan-Meier method and the Cox proportional hazards model were used to assess the VTE risk.
\end{abstract}

Results: The 5-year cumulative risk for VTE was significantly higher in the cervical cancer group than in the control group $(3.3 \%$ vs $0.3 \%, p<0.001)$. The hazard ratio for VTE was 10.14 times higher in the cervical cancer group than in the controls. The combined presence of more comorbidities was associated with a higher risk for VTE. Furthermore, cervical cancer patients without VTE had a significantly higher survival $(75.3 \%$ vs $30.3 \%, p<0.001)$.

Conclusions: The cumulative risk of VTE was significantly higher in cervical cancer patients, and these patients also had lower survival rates. Strategies to reduce these risks need to be examined.

\section{Background}

Cervical cancer remains the most important malignant disease in women, with an age-adjusted incidence of 26.2 per one hundred thousand people in Taiwan.[1] Venous thromboembolism (VTE) is a life-threatening condition in cervical cancer. Its incidence can be stratified by patient, tumor, or treatment. Reports of the VTE incidence in cervical cancer vary, and range from $0 \%$ to 34\%.[2] For cancer-related VTE, the incidence of VTE is higher in the first few months after cancer is diagnosed, and subsequently decreases with time.[3,4] A literature review indicates that surgery, chemotherapy, or radiotherapy can be risk factors for VTE.[5] Radiationinduced vascular disease has been reported. Concurrent

\footnotetext{
* Correspondence: oncology158@yahoo.com.tw

${ }^{\dagger}$ Equal contributors

'Department of Radiation Oncology, Buddhist Dalin Tzu Chi General Hospital, Chiayi, Taiwan

${ }^{5}$ School of Medicine, Tzu Chi University, Hualien, Taiwan

Full list of author information is available at the end of the article
}

chemotherapy and radiotherapy is the standard treatment for patients with advanced cervical cancer, and it increases survival.[6] Although the number of long-term survivors has increased, data on the incidence of radiotherapy- or chemotherapy-induced VTE remains limited. Thus, the aim of this study was to evaluate the incidence of VTE in cervical cancer patients during a 5year follow-up.

\section{Materials and methods}

The study analyzed 2003-2008 data from the National Health Insurance Research Database (NHIRD), provided by the National Research Institutes in Taiwan. The NHIRD contains the medical benefit claims for $97 \%$ of the population from a registry of board-certified physicians and contracted medical facilities. The procedures we followed were in accordance with the ethical standards of the committee on human experimentation of our institution and with the Helsinki Declaration. This study was approved by the Institutional Review Board at

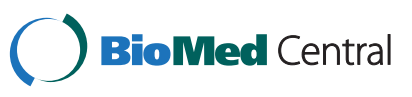


Buddhist Dalin Tzu Chi General Hospital and approved number is B10001017.

The study included two cohorts. The principal diagnosis in the first cohort was cervical cancer, identified by the International Classification of Disease, Ninth Revision, Clinical Modification (ICD-9-CM) code 180. The different treatment modalities included surgery alone (S), radiotherapy alone (RT), surgery plus radiotherapy (SRT), surgery plus chemotherapy and radiotherapy (SCRT), surgery plus chemotherapy (SCT), chemotherapy and radiotherapy (CCRT), and chemotherapy alone (CT). Participants in the second cohort, who served as a control group, underwent appendectomy (ICD-OP code 47). Appendectomy patients were selected as a control group because of their similarity to the general population. $[7,8]$

Data on each patient were collected starting from the first hospitalization or outpatient visit in 2003. Totally, 1013 cervical cancer patients after treatment and 2026 appendectomy patients were eligible. Because of significant differences in mean age and comorbidities between the 2 groups, the control cohort criteria were further refined by randomly selecting 1013 matched female appendectomy patients at 1: 2 ratio based on age and comorbidities.

The primary dependent variable was venous thromboembolism (VTE; ICD-9-CM codes 415.11,415.19,451.11, $451.19,451.81,453.40,453.41,453.42,4538-4539)$. In both

Table 1 Demographic characteristics and comorbidities of the cervical cancer and control groups

\begin{tabular}{|c|c|c|c|}
\hline Variable & Cervical cancer group $(\mathrm{N}=1,013)$ No. $(\%)$ & Control group(N= 2,026)No. (\%) & $p$ \\
\hline Age, year & & & $<0.001$ \\
\hline$\leqq 44$ & $366(36.1)$ & $641(31.6)$ & \\
\hline $45-54$ & $325(32.1)$ & $556(27.4)$ & \\
\hline $55-64$ & $155(15.3)$ & $351(17.3)$ & \\
\hline $65-74$ & $130(12.8)$ & $325(16.0)$ & \\
\hline$\geqq 75$ & $37(3.7)$ & $153(7.6)$ & \\
\hline Hypertension & & & $<0.001$ \\
\hline Yes & $256(25.3)$ & $395(19.5)$ & \\
\hline No & $757(74.7)$ & $1631(80.5)$ & \\
\hline Diabetes & & & 0.22 \\
\hline Yes & $130(12.8)$ & $229(11.3)$ & \\
\hline No & $883(87.2)$ & $1797(88.7)$ & \\
\hline Coronary heart disease & & & $<0.001$ \\
\hline Yes & $92(9.1)$ & $90(4.4)$ & \\
\hline No & $921(90.9)$ & $1936(95.6)$ & \\
\hline Hyperlipidemia & & & $<0.001$ \\
\hline Yes & $36(3.6)$ & $30(1.5)$ & \\
\hline No & $977(96.4)$ & 1996 (98.5) & \\
\hline Geographic region & & & 0.008 \\
\hline Northern & $444(43.8)$ & $942(46.5)$ & \\
\hline Central & $289(28.5)$ & $496(24.5)$ & \\
\hline Southern & $262(25.9)$ & $518(25.6)$ & \\
\hline Eastern & $18(1.8)$ & $70(3.5)$ & \\
\hline Urbanization level & & & 0.06 \\
\hline Urban & $268(26.5)$ & $618(30.5)$ & \\
\hline Suburban & $464(45.8)$ & $870(42.9)$ & \\
\hline Rural & $281(27.7)$ & $538(26.6)$ & \\
\hline EC & & & 0.01 \\
\hline EC 1,2 & $384(37.0)$ & $836(41.3)$ & \\
\hline EC 3 & $442(43.6)$ & $897(44.3)$ & \\
\hline EC 4 & $187(18.5)$ & $293(14.5)$ & \\
\hline
\end{tabular}


groups, subjects who suffered a VTE and diagnosed before the index date were excluded from the data analysis. Patients were also excluded if distant metastases were diagnosed at initial.

Deaths recorded in the database were marked to calculate the vascular event-free survival, with cases censored if the patients died from non-vascular causes during follow-up. The independent variables were age, comorbidities, geographic region, urbanization level, and socioeconomic status. Comorbidities included hypertension, diabetes, coronary heart disease, and hyperlipidemia. There were four geographic regions (Northern, Central, Southern, and Eastern) and three urbanization levels (urban, suburban, and rural). This study also used enrollee category (EC) as a proxy measure for the socioeconomic status. All patients were categorized as EC1 (the highest socioeconomic status), EC2, EC3, or EC4 (the lowest socioeconomic status). These variables were associated with vascular disease.[9-11]

\section{Statistical analysis}

The statistical software packages SAS (version 9.2; SAS Institute, Inc., Cary, NC, USA) and SPSS (version 17; SPSS Inc., Chicago, IL, USA) were used for data analysis. Inter-cohort differences in the frequency of variables were evaluated using the chi-square test. Cox regression model analysis was used to calculate the effects of VTE events on the case and control groups after adjusting for confounders. The risk factors included age, comorbidities, geographic region, urbanization level, and socioeconomic status. The vascular event-free survival was calculated using the Kaplan-Meier method. $P<0.05$ was defined as statistically significant.

\section{Results}

The distribution of demographic characteristics and comorbidities for the two cohorts is shown in Table 1. Compared to the control group (after matching), the case group had a high prevalence of hypertension, coronary heart disease, hyperlipidemia, and diabetes. There were $321,162,246,69,43,36$, and 624 patients in the RT, SRT, CCRT, SCRT, SCT, CT, and S groups, respectively. The 5-year cumulative risk of VTE in the RT, SRT, CCRT, SCRT, SCT, CT, and S groups was $3.0 \%, 3.8 \%$, $3.0 \%, 3.0 \%, 6.5 \%, 11.0 \%$, and $2.1 \%$, respectively. If we excluded chemotherapy alone, there were no significant differences in the prevalence of vascular events between different treatment modalities. In addition, the 6 months, 1 year and 5 year cumulative risk of VTE in all case groups were $0.5 \%, 1.4 \%$ and $2.9 \%$, respectively.

At the end of follow-up in 2008, a total of 39 patients had VTE, including 33 in the cervical cancer group and 6 in the control group. The median interval between treatment and the VTE event was 21.4 months. The average range for follow-up duration was 66.5 months. The 5-year cumulative risk of VTE was significantly higher for the cervical cancer group than for the controls ( $3.3 \%$ vs $0.3 \%, p<0.001$; Figure 1$)$. Figure 2 shows survival in cervical cancer patients by comparing the vascular event of VTE. Cervical cancer patients without VTE had significantly higher survival $(75.3 \%$ vs $30.3 \%$, $p<0.001$; Figure 2).

Unadjusted and adjusted hazard ratios for the association of VTE with cervical cancer after treatment and appendectomy are shown in Table 2. After adjustments for age and comorbidities, the hazard ratio for VTE during the 5-year follow-up was 10.14 times higher than among controls.

Five risk factors (age older than 55 years, hypertension, diabetes, coronary artery disease, and hyperlipidemia) were used to stratify the cervical cancer cohort into 2 groups: a low-risk group ( $\mathrm{n}=276$; no risk factors), and high-risk group ( $\mathrm{n}=737 ; \geq 1$ risk factor). The 5 -year $\mathrm{cu}$ mulative risks of VTE in the stratified groups were $2.2 \%$ and $3.4 \%$, respectively (Table 3 ).

\section{Discussion}

Cervical cancer is an important health problem. Although its incidence is decreasing, it remains the most important cause of cancer death in women from Taiwan. VTE is a life-threatening condition in cervical cancer. It is important to record the incidence of VTE and design preventive strategies. For all cervical cancer patients, the 5-year cumulative risk of VTE was $3.3 \%$. Our study reveals that cervical cancer patients have a higher cumulative risk of VTE as compared to the general population.

The incidence of VTE in cervical cancer can be stratified into patient, tumor, or treatment-related. However,

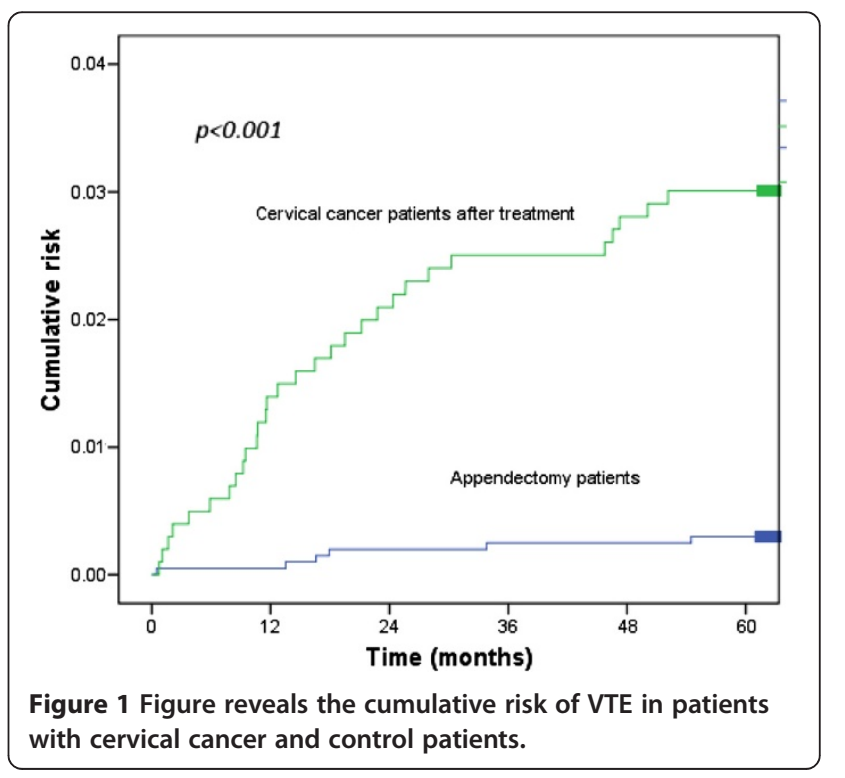




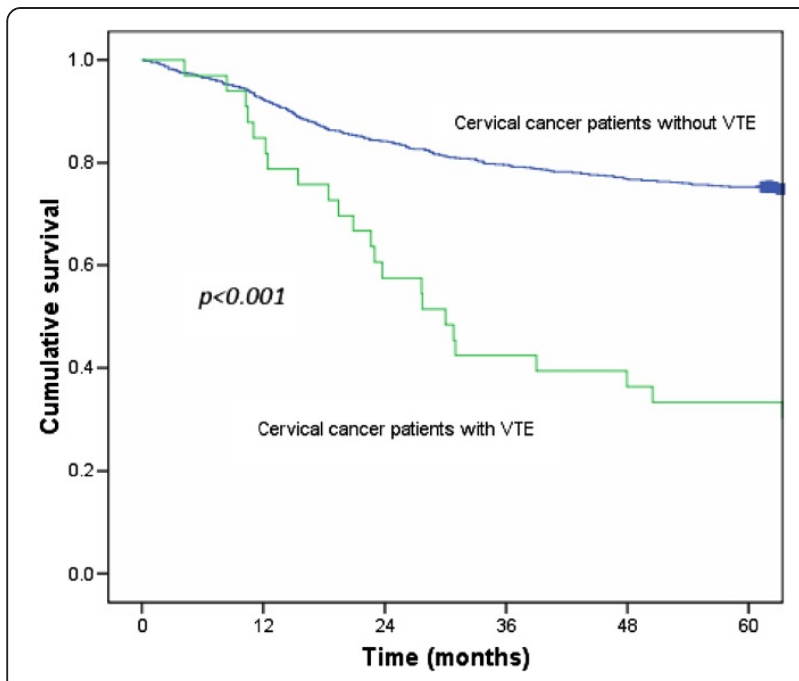

Figure 2 The survival of cervical cancer patients with VTE compared with survival in patients without VTE.

it is difficult to separate these three categories. Few reports described the risk factors for patients with cervical-cancer-related VTE, which include age[12], comorbidities[13], immobilization[14], or inherited traits [15]. However, these reports were not recorded specifically in patients with cervical cancer.[2] For cancerrelated VTE, Chew et al. reported the incidence of VTE from cancer diagnosis data. The VTE rate of uterus at one year was 1.6\%.[16] Furthermore, the stage of the disease is defined by tumor extension and the extent to which it influences venous compression and stasis. Importantly, clinical findings reported an increased VTE risk in the advanced stages.[17] This means that the incidence of VTE correlates with the biological aggressiveness of the tumor.[18] Our study had similar reports, and cervical cancer patients without VTE experienced significantly higher survival rates.

Compared with the general population, cancer patients are often observed to have lower socioeconomic status. [19] This has subsequently been associated with a higher prevalence of comorbidities, such as diabetes mellitus, hypertension, or hyperlipidemia. These factors exacerbate vascular disease. In our study, five risk factors were used to stratify the cancer patients into low- and highrisk groups. The 5-year VTE incidence was lower in the low-risk group, $2.2 \%$, than in the high-risk groups, $3.4 \%$. Patients with more comorbidities had a higher risk of
Table 3 The annual VTE cumulative risk in different stratified groups

\begin{tabular}{lll}
\hline $\mathbf{N}$ of Risk Factors & VTEevents, $\mathbf{N}(\%)$ & 5-yearVTErisk (\%) \\
\hline $0(\mathrm{~N}=276)$ & $7(2.5)$ & 2.2 \\
$1(\mathrm{~N}=737)$ & $26(3.5)$ & 3.4 \\
\hline
\end{tabular}

Risk factors included age older than 55 years, hypertension, diabetes, coronary artery, disease, and hyperlipidemia.

VTE. Therefore, interventions aimed at VTE prevention are extremely important. Complete surveys of modifiable risk factors and intensive lifestyle modification are recommended in patients with multiple comorbidities. In addition, some studies reported that low-molecularweight heparin $(\mathrm{LMWH})$ for thromboprophylaxis shows benefits for patient survival.[20] Further studies are recommended to determine the role in primary prevention of VTE.

Several limitations of this study should be mentioned. First, during data collection, the recording of VTE events in the NHIRD may have been inadvertently missed. In a multivariate analysis, the increased incidence of VTE was unrelated to the addition of platinum-based chemotherapy to radiotherapy. The relatively small size of the census populations and the relatively short follow-up period probably hindered the analysis. Second, the NHIRD is used primarily for administrative purposes and does not provide information on clinical characteristics, including staging, VTE severity, and biochemical data. Consequently, these pieces of data were not available for analysis in this study. Third, the NHIRD database does not have information on tobacco use, dietary habits, and body mass index, which may be additional risk factors for VTE. Forth, the vascular events in cervical cancer are low (only 33 events from 2003-2008), we preferred to use whole cervical cancer population to match control group. Although appendicitis is subject to Berkson's bias, there are evidences that appendectomy patients could be selected as a control group because of their similarity to the general population. $[7,8,21]$ Appendectomy patients are an acceptable comparator. Take together, given the magnitude and statistical significance of the effects observed in this study, these limitations are unlikely to alter our conclusion.

\section{Conclusion}

In this cohort study, the cumulative risk of VTE was significantly higher in cervical cancer patients than in the general population. Cervical cancer patients with VTE

Table 2 Crude and adjusted hazard ratios for different vascular events in the $\mathbf{5}$-year follow-up period

\begin{tabular}{|c|c|c|c|c|c|c|}
\hline & & Events(\%) & UnadjustedHR (95\%Cl) & $p$ & AdjustedHR $(95 \% \mathrm{Cl})$ & $p$ \\
\hline \multirow[t]{2}{*}{ VTE } & Control group $(N=2026)$ & $6(0.3)$ & 1 & & 1 & \\
\hline & Cervix cancer group $(N=1013)$ & $33(3.3)$ & $10.87(4.54-25.99)$ & $<0.001$ & $10.14(4.19-24.54)$ & $<0.001$ \\
\hline
\end{tabular}

Adjusted for age, hypertension, diabetes, coronary heart disease, hyperlipidemia, geographic region, urbanization level, and enrollee category. VTE indicates venous thromboembolism. 
had significantly lower survival rates. Strategies to reduce these risks need to be examined.

\section{Acknowledgment}

This study utilizes data from the National Health Insurance Research Database provided by the Bureau of National Health Insurance, Department of Health, and managed by National Health Research Institutes (registry number 99029). The interpretation and conclusions contained herein are not those of the Bureau of National Health Insurance, Department of Health, or National Health Research Institutes

\section{Author details}

'Department of Radiation Oncology, Buddhist Dalin Tzu Chi General Hospital, Chiayi, Taiwan. ${ }^{2}$ Department of Chinese Medicine, Buddhist Dalin Tzu Chi General Hospital, Chiayi, Taiwan. ${ }^{3}$ Department of Otolaryngology, Hematology, Buddhist Dalin Tzu Chi General Hospital, Chiayi, Taiwan. ${ }^{4}$ Department of Oncology, Buddhist Dalin Tzu Chi General Hospital, Chiayi, Taiwan. ${ }^{5}$ School of Medicine, Tzu Chi University, Hualien, Taiwan.

\section{Conflict of interest}

The authors declare no conflicts of interest.

\section{Authors' contributions}

TSJ, RYX and HSK developed the ideas for these studies, performed much of the work, and drafted the manuscript. TSJ, CWY and HSK revised the manuscript. LCC, LMS, SYC, CWY and LHY designed the study, managed and interpreted the data. TSJ and HFC performed the statistical analysis. All authors read and approved the final manuscript.

Received: 14 November 2011 Accepted: 21 June 2012

Published: 21 June 2012

\section{References}

1. Chen YY, You SL, Chen CA, Shih LY, Koong SL, Chao KY, Hsiao ML, Hsieh CY, Chen CJ: Effectiveness of national cervical cancer screening programme in Taiwan: 12-year experiences. Br J Cancer 2009, 101:174-177.

2. Barbera $\mathrm{L}$, Thomas $\mathrm{G}$ : Venous thromboembolism in cervical cancer. Lancet Oncol 2008, 9:54-60.

3. Maduro JH, den Dekker HA, Pras E, de Vries EG, van der Zee AG, Klokman WJ, Reyners AK, van Leeuwen FE, Langendijk JA, de Bock GH, Gietema JA: Cardiovascular morbidity after radiotherapy or chemoradiation in patients with cervical cancer. Int J Radiat Oncol Biol Phys 2010, 78:1337-1344

4. Al-Badawi IA, Al-Aker M, AlSubhi J, Salem H, Abduljabbar A, Balaraj K, Munkarah A: Laparoscopic ovarian transposition before pelvic irradiation: a Saudi tertiary center experience. Int J Gynecol Cancer 2010, 20:10821086.

5. Jacobson G, Lammli J, Zamba G, Hua L, Goodheart MJ: Thromboembolic events in patients with cervical carcinoma: Incidence and effect on survival. Gynecol Oncol 2009, 113:240-244.

6. Green JA, Kirwan JM, Tierney JF, Symonds P, Fresco L, Collingwood M, Williams CJ: Survival and recurrence after concomitant chemotherapy and radiotherapy for cancer of the uterine cervix: a systematic review and meta-analysis. Lancet 2001, 358:781-786.

7. Lin HC, Tsai SY, Lee HC: Increased risk of developing stroke among patients with bipolar disorder after an acute mood episode: a six-year follow-up study. J Affect Disord 2007, 100:49-54.

8. Lin HC, Tsai SY, Lee HC: No higher risk of myocardial infarction among bipolar patients in a 6-year follow-up of acute mood episodes. Psychosom Med 2008, 70:73-76.

9. Chen CY, Liu CY, Su WC, Huang SL, Lin KM: Factors associated with the diagnosis of neurodevelopmental disorders: a population-based longitudinal study. Pediatrics 2007, 119:e435-e443.

10. McFadden $E$, Luben $R$, Wareham $N$, Bingham $S$, Khaw KT: Social class, risk factors, and stroke incidence in men and women: a prospective study in the European prospective investigation into cancer in Norfolk cohort. Stroke 2009, 40:1070-1077.

11. Lin HC, Lin YJ, Liu TC, Chen CS, Chiu WT: Urbanization and stroke prevalence in Taiwan: analysis of a nationwide survey. J Urban Health 2007, 84:604-614.
12. Silverstein MD, Heit JA, Mohr DN, Petterson TM, O'Fallon WM, Melton LJ 3rd: Trends in the incidence of deep vein thrombosis and pulmonary embolism: a 25-year population-based study. Arch Intern Med 1998, 158:585-593.

13. Heit JA, Silverstein MD, Mohr DN, Petterson TM, O'Fallon WM, Melton LJ 3rd: Risk factors for deep vein thrombosis and pulmonary embolism: a population-based case-control study. Arch Intern Med 2000, 160:809-815

14. Samama MM: An epidemiologic study of risk factors for deep vein thrombosis in medical outpatients: the Sirius study. Arch Intern Med 2000, 160:3415-3420.

15. Wang X, Fu S, Freedman RS, Kavanagh JJ: Venous thromboembolism syndrome in gynecological cancer. Int J Gynecol Cancer 2006, 16 (Suppl 1):458-471.

16. Chew HK, Wun T, Harvey D, Zhou H, White RH: Incidence of venous thromboembolism and its effect on survival among patients with common cancers. Arch Intern Med 2006, 166:458-464.

17. Sallah S, Wan JY, Nguyen NP: Venous thrombosis in patients with solid tumors: determination of frequency and characteristics. Thromb Haemost 2002, 87:575-579.

18. Wun T, White RH: Epidemiology of cancer-related venous thromboembolism. Best Pract Res Clin Haematol 2009, 22:9-23.

19. van der Aa MA, Siesling S, Louwman MW, Visser O, Pukkala E, Coebergh JW: Geographical relationships between sociodemographic factors and incidence of cervical cancer in the Netherlands 1989-2003. Eur J Cancer Prev 2008, 17:453-459.

20. Einstein MH, Pritts EA, Hartenbach EM: Venous thromboembolism prevention in gynecologic cancer surgery: a systematic review. Gynecol Oncol 2007, 105:813-819.

21. Feinstein AR, Walter SD, Horwitz RI: An analysis of Berkson's bias in casecontrol studies. J Chronic Dis 1986, 39:495-504.

doi:10.1186/1756-0500-5-316

Cite this article as: Tsai et al:: The incidence of venous

thromboembolism in cervical cancer: a nationwide population-based study. BMC Research Notes 2012 5:316.

\section{Submit your next manuscript to BioMed Central and take full advantage of:}

- Convenient online submission

- Thorough peer review

- No space constraints or color figure charges

- Immediate publication on acceptance

- Inclusion in PubMed, CAS, Scopus and Google Scholar

- Research which is freely available for redistribution 